\title{
Programa pedagógico interactivo para la reforestación ambiental de la EUN Policarpa Salavarrieta ${ }^{1}$
}

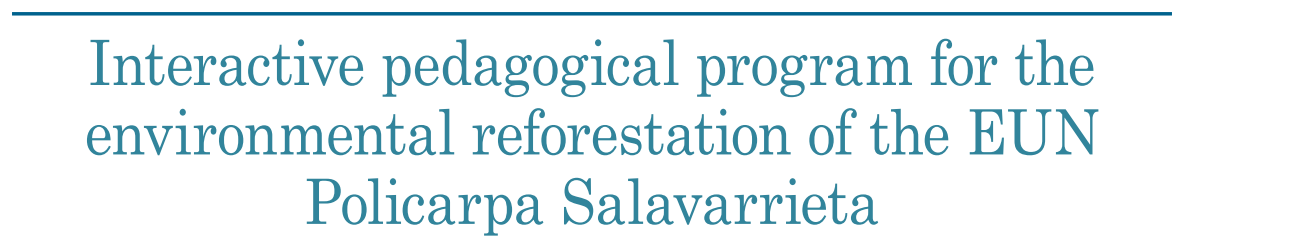

DOI: http://dx.doi.org/10.17981/cultedusoc.9.2.2018.14

Fecha de recepción: 23/06/2018. Fecha de aceptación: 18/09/2018

Martha Suarez Prieto ${ }^{2}$

IED de Básica y media de Concordia, sede EUN Policarpa Salavarrieta (Colombia)

marpeme17@yahoo.es

Para citar este artículo

Suarez, M. (2018). Programa pedagógico interactivo para la reforestación ambiental de la EUN Policarpa Salavarrieta. Cultura. Educación y Sociedad 9(2), 147-158. DOI: http://dx.doi.org/10.17981/cultedusoc.9.2.2018.14

\section{Resumen}

Se hace la descripción de un programa pedagógico, que permita la reforestación ambiental de la Institución Educativa, Policarpa Salavarrieta, de acuerdo al modelo pedagógico institucional, bajo los referentes teóricos de Perdomo, A. \& Díaz, W. (2015), (Soto, 2011), (Macgregor,\& Ortega, 2013). Se asume un abordaje cualitativo, se tomó como muestra 21 estudiantes del grado cuarto. Los instrumentos de recolección de información fueron la observación directa, registrada en un diario de campo y la entrevista estructurada. Dentro de los hallazgos encontrados, es importante destacar los conocimientos generales de los estudiantes sobre la degradación ambiental, y así mismo se evidencian conocimientos para realizar procesos de reforestación, aunque falta apropiación que permitan armonizar un programa ambiental desde la IEP, articulado con las diferentes asignaturas.

Palabras clave: Programa pedagógico, reforestación ambiental, articulación.

\begin{abstract}
The description of a pedagogical program is made, which allows the environmental reforestation of the Educational Institution, Policarpa Salavarrieta, according to the institutional pedagogical model, under the theoretical referents of Perdomo, A. \& Diaz, W. (2015), (Soto, 2011), (Macgregor, \& Ortega, 2013). A qualitative approach is assumed, 21 students from the fourth grade were taken as a sample. Among the findings found, it is important to highlight students' general knowledge about environmental degradation, and they also demonstrate knowledge to carry out reforestation processes, although there is a lack of appropriation to harmonize an environmental program from the IEP, articulated with the different subjects.
\end{abstract}

Keywords: Pedagogical program, environmental reforestation, articulation.

\footnotetext{
${ }^{1}$ Este artículo ha sido derivado del Programa de Fortalecimiento de la Cultura Ciudadana y Democrática CT+I a través de la IEP apoyada en TIC en el Departamento de Magdalena: CICLÓN. Desarrollado con docentes miembros del Grupo de investigación "Cuidando mi medio ambiente" pertenecientes a la Institución Educativa Departamental de Básica y media de Concordia, sede EUN Policarpa Salavarrieta.
}

${ }^{2}$ Líder del grupo de investigación "Cuidando mi medio ambiente". 


\section{Introducción}

Actualmente, la educación, demanda herramientas didácticas - pedagógicas en donde los estudiantes vivan las necesidades reales de su contexto y además se desenvuelvan asumiendo nuevos roles, articulando el conocimiento, la disciplina y la cultura ambiental, bajo estrategias educativas.

De acuerdo a las intenciones del estudio, se requiere de un programa pedagógico que permita la reforestación ambiental, y que promueva al interior de la institución educativa la participación activa de estudiantes, docentes y comunidad en General.

La Reforestación resulta una de las prioridades de la IED Policarpa Salavarrieta, la cual surge como una estrategia para resguardar el entorno, permitiendo así que el conocimiento y compromiso ambiental sea más natural por medio del ejercicio continuo y la interacción del individuo con el ambiente, al mismo tiempo provocando el razonar e involucrando los valores y dando paso a que en los estudiantes haya actitudes para describir, comprender, asociar, apreciar y transformar su contexto.

El objetivo del presente estudio es crear un programa pedagógico para la reforestación ambiental de la EUN Policarpa Salavarrieta en el Municipio de Concordia, en el Departamento del Magdalena.

La grave situación que experimenta el planeta hace imperante que se refuercen competencias ambientales en los estudiantes y esto solo puede lograrse utilizando estrategias pedagógicas innovadoras como la arborización y reforestación, y también dando uso a los instrumentos educativos que creen operaciones concretas orientadas a la solución de problemas ambientales que existan en la práctica cuotidiana. Tal como lo expresa Perdomo, A. \& Díaz, W. (2015). Los árboles cumplen un beneficio en la naturaleza; de tal modo las actividades derivadas de la misma deben estar enca- minadas g hacia la preservación del medio ambiente para que las futuras generaciones puedan contar con un entorno sano y un mundo más verde.

Desde los años 90 en países latinoamericanos, se ha visto la necesidad de poner cara a la problemática medioambiental, pues es en países como Perú, Ecuador, Chile, Colombia reconocen los estragos del crecimiento desordenado, a causa de una inadecuada planificación y uso de los recursos naturales. (Soto, 2011). Argentina, por ejemplo, ha tenido en los últimos 15 años un impacto medioambiental fuerte debido al acrecentamiento de la superficie edificada, la desintegración del paisaje, la lesión constante e irreversible de suelo con alta aptitud agrícola y el acrecimiento de tiraderos de basura al aire libre . (Macgregor, \& Ortega, 2013).

Por su parte Colombia, es un país rico en flora y fauna, pero es notorio así mismo observar los desastres medio ambientales que se presentan en el diario vivir, factor que el hombre ha ocasionado por la poca consciencia que existe sobre la manipulación y uso indebido de lo que el entorno ofrece. La tala de árboles aumenta constantemente, como formas de consumo y adquisición de materia prima para la elaboración de instrumentos de necesidades diarias. (Stern, P. C., Dietz, T. \& Kalof, L. 1993).

La ausencia de árboles se ha convertido en una problemática de abordaje especial, es en el Departamento del Magdalena, y en especial, en las Instituciones Educativas del mismo, que surge la necesidad de diseñar programas que permitan crear ambientes lo más natural posibles, incrementar las zonas verdes y enriquecer los corredores educativos.

Esta situación no sólo compete a especialistas en asuntos tecnológicos, biológicos y científicos; también es la consecuencia de los comportamientos de las personas, quienes en su mayoría tienden a alejarse de la 
situación y sienten parte de la situación e identifican las acciones que pueden emprender como individuos para lograr cambios, (Herrera, 2014, citando a Martínez-Soto, 2006).

\section{Conceptualización de Reforestación}

La reforestación puede ser definida por el conjunto de tareas que tiene en cuenta la planeación, la operación y el control y vigilancia de todas las actividades en directa relación la plantación de árboles. (Manual básico de Prácticas de reforestación; 2010).

Por otro lado, El manual básico de Prácticas de reforestación, (2010) citando la Ley General de Desarrollo Forestal Sustentable (LGDFS). (s.f) definen que la reforestación como "el establecimiento inducido de vegetación forestal en terrenos forestales.

Existen dos tipos de reforestación, la urbana y la rural. La primera; la que se establece dentro de las ciudades con diversos fines u objetivos y la segunda; es la que con base a los objetivos se establece en superficies forestales potencialmente forestales donde en un principio existían bosques, selvas o vegetación semiárida. (El manual básico de Prácticas de reforestación, 2010, citando la Ley General de Desarrollo Forestal Sustentable (LGDFS). (s.f)).

Autores como Bloomfield \& Calle (s.f). Definen la reforestación como "la reforestación es un método activo que busca recuperar la cobertura de bosque en un sitio deforestado mediante la introducción de semillas o plántulas".

\section{Arborización como opción sustentable}

Los arboles constituyen los elementos más singulares de los ecosistemas, todo, por el sinnúmero de funciones ecológicas que realizan y la estabilidad que aportan a los mismos. (Fundación Gondwana para el desarrollo sostenible s.f).
Los árboles resguardan de los rayos solares, limpian la luz intensa y adornan el entorno, pero, sobre todo, "tienen una función de regulación bioclimática que beneficia la humedad del viento al absorber el agua por sus raíces y restituirla por evapotranspiración" (Reyes \& Gutierrez 2010).

Reyes \& Gutierrez (2010) citando a Lizana (2003) \& Sorensen (1998) señalan que otro de los beneficios de los arboles tiene que ver con la radiación solar, ya que por medio de la sombra que ofrece este es capaz de absorber calor y, en esa medida, controlar la temperatura, ellos causan efectos significativos al momento de hacer su implantación. (Tamayo, 1993 y Toledo y González, 2007).

Por otro lado, según Vining, J. \& Ebreo, A. (1992). Si existiera un debido orden en la preservación de las especies, y también en el bienestar físico y psíquico del ser humano; y de igual forma en la plantación y conservación de árboles, todos seriamos beneficiados siendo nosotros el suministro de sombra y oxígeno, se les restara al calor, a los vientos y al ruido, el control de la persistencia micro climática, así como el combate a la erosión y el abastecimiento de abrigo y alimento para la fauna. (Reyes \& Gutierrez, 2010).

Según la tipología de árboles que se desean plantar, resulta fundamental resaltar que el aporte de nutrientes dependerá de las características de cada especie (leguminosas, caducifolio, contenido de nutrientes en sus hojas, sistema radicular, degradación de la hojarasca, etc.). La autoridad responsable necesita ser capaz de contar con la experiencia técnica necesaria para integrar variables ambientales y técnicas en acciones de arborización. (Chávez s.f).

Con el paso de los años se ha notado fuertemente un accionar despreocupa- 
do, egoísta y contaminante por parte del hombre sobre los recursos, sin embargo; actualmente se ha hecho consciente que los daños causados afectan directamente la el accionar y las relaciones humanas. Es por esto que se puede inferir que los pensamientos sociales, culturales, económicos han permitido trascender de conductas que anteriormente dominaban el comportamiento de los individuos. (Acuña \& Gil; 2014).

Es importante científica y académicamente comprender el papel de los factores conductuales y mentales implicados en la relación del hombre con la naturaleza es esencial para encontrar la solución al problema medioambiental (Ramirez \& Del La Hoz; 2017, citando a Bravo, 2010), ya que se necesita conocer como asumen los humanos el problema ambiental.

Esta información sería absolutamente útil para crear modelos enfocados a explicar por qué y de qué manera las personas realizan determinadas conductas en relación con el ambiente, además de diseñar modelos explicativos de comportamientos ambientales dentro de la empresa y cómo son asumidos por los trabajadores. (Ramirez \& Del La Hoz; 2017)

De esta manera, se plantea la necesidad de realizar cambios actitudinales en los seres humanos, que conduzcan a la generación de modelos y patrones de conducta más acordes con el cuidado del Medio Ambiente partiendo desde su ciclo de interacción vital, a pesar de que autores como González (2005), afirman que la atribución de la degradación ambiental se externaliza al contexto global, sin contemplar la experiencia personal como parte del compromiso en el fortalecimiento de conductas pro ambientales. (Herrera, 2014).

Según Herrera, (2014); citando a (Schwartz, 1992) "en la actualidad se está teniendo en cuenta un modelo para el estudio de la relación de los valores personales con el comportamiento pro ambiental es el de "La estructura y contenido universal de los valores humanos". En él se clasifican una serie de contenidos que comparten los valores, a manera de objetivos que motivan a la acción, los cuales se orientan bajo dos dimensiones: Conservadurismo (seguridad, conformidad, tradición) Vs apertura al cambio (autodirección, hedonismo, estimulación) y autotrascendencia (benevolencia, universalismo) Vs autopromoción (logros, poder).

Hacia 1993, Stern, Dietz y Kalof, realizaron un estudio en el que se asume que la preocupación ambiental incluye un conjunto de sentimientos personales de normas u obligación que conducen al establecimiento de conductas pro-ambientales, siempre y cuando el individuo posea creencias relacionadas con que las condiciones medioambientales tienen efectos negativos para el mismo individuo, su entorno y las demás especies que lo habitan. (Weigel, R. \& Weigel, J. (1978); Zeithaml, C. y Zeithaml, V. (1984); Zeithaml, C. y Zeithaml, V.; (1984). Zikmund, W. y Stanton, W. (1971).

Así mismo, González y Amérigo (1999) plantean que la preocupación y comportamiento pro ambiental pueden ser explicados siempre y cuando se tengan presentes los valores personales, como motivadores de conductas.

Según Herrera, (2014) citando a Berenguer (1998) la investigación en el comportamiento pro ambiental se ha desarrollado teniendo en cuenta los tipos de motivación del ser humano. Según el Ministerio De Educación Nacional (2014) el objetivo primordial es mejorar la calidad educativa, y para ello han diseñado programas que buscan la transformación de los escenarios en donde se propende la enseñanza- aprendizaje. 


\section{Metodología}

La investigación es de tipo cualitativa, el alcance es descriptivo, por lo que permite identificar tipologías al objeto de estudio, marca pautas y maneras a conducir del universo estudiado, constituye conductas concretas y revela y evidencia la reunión entre categorías de búsqueda. Conforme a las propósitos, el estudioso señala el tipo de descripción que se plantea a realizar. (Martínez 2006).

\section{Escenario y actores}

Los participantes del estudio fueron los estudiantes de la Institución Educativa Básica y media de Concordia, sede EUN Policarpa Salavarrieta, con una representación de (30) alumnos del grado cuarto.

\section{Técnicas e instrumentos de recolección de la información}

Dado el tipo de investigación y las características del escenario de investigación se decide utilizar como técnicas de recolección: La entrevista. De manera que la información es registrada en un instrumento de entrevista estructurada, que permiten su clasificación y nos proporciona facilidad para su análisis e interpretación. (Sellitz, C. 1994).

\section{Procedimiento.}

La ejecución de la presente investigación está enmarcada en los siguientes momentos:

Momento (1): Elección del fenómeno a estudiar.

Momento (2): Hacer el sondeo diagnóstico de los objetos de interés.

Momento (3): Definición de las categorías de estudio: Programa pedagógico, reforestación ambiental.
Momento (4): Definición de los instrumentos de recolección de la información. Para el diseño de la entrevista estructurada Se construyeron los contenidos de acuerdo de acuerdo a la problemática estudiada. Luego fue enviada a pares expertos para su respectiva validación.

El diseño de las preguntas, tuvo una guía que permitiera elaboración definitiva del cuestionario y del plan operativo de la entrevista basándose en propósitos específicos de la investigación, se realizó una validación presentada. (Ramírez, L., Arcilla, A., Buriticá, L. y Castrillón, J. 2004).

Una vez validados los contenidos se elaboró y diseñó un instrumento para ser sometido a una prueba piloto, tomando los aportes más significativos de los aspectos evaluados. Culminada la prueba piloto se envió de nuevo a los jueces expertos, quienes hicieron las correcciones y recomendaciones pertinentes. Finalmente se procede a realizar la aplicación a la población objeto de estudio.

Momento (5): Aplicación de la entrevista estructurada y los registros de observación.

Momento (6): Sistematización de la información a la luz de los datos recolectados y articulación con la teoría.

Momento (7): Generación de resultados. Momento (8): Socialización de los hallazgos encontrados.

\section{Resultado}

A continuación, se presentan lo hallazgos derivados de los instrumentos aplicados, (entrevista estructurada). Y diario de campo. Para estos hallazgos se tuvo en cuenta la pregunta orientadora. ¿De qué manera la creación de un programa pedagógico en la EUN Policarpa Salavarrieta permite la reforestación ambiental? 


\begin{tabular}{|c|c|c|c|}
\hline $\begin{array}{l}\text { Categoría } \\
\text { de estudio }\end{array}$ & $\begin{array}{c}\text { Pregunta } \\
\text { orientadora }\end{array}$ & $\begin{array}{l}\text { Discurso de } \\
\text { los actores }\end{array}$ & $\begin{array}{c}\text { Articulación y } \\
\text { sistematización teórica }\end{array}$ \\
\hline $\begin{array}{l}\text { Reforestación } \\
\text { ambiental }\end{array}$ & $\begin{array}{l}\text { ¿Alguna vez } \\
\text { ha sembrado } \\
\text { árboles? } \\
\text { ¿Cómo se } \\
\text { ha sentido } \\
\text { haciéndolo? }\end{array}$ & $\begin{array}{l}\text { "si he sembrado árboles } \\
\text { y me he sentido muy } \\
\text { feliz porque cuido la } \\
\text { naturaleza "Si, porque } \\
\text { estoy ayudando a la } \\
\text { naturaleza" "si porque } \\
\text { me gusta ayudar el } \\
\text { medio ambiente" "si } \\
\text { he sembrado arboles } \\
\text { con cuidado y me he } \\
\text { sentido muy bien porque } \\
\text { ayudo a la naturaleza", } \\
\text { "bien porque ayudo } \\
\text { a la naturaleza y } \\
\text { a la institución”," } \\
\text { feliz porque me gusta } \\
\text { cuidar el ambiente", " } \\
\text { me he sentido alegre } \\
\text { y feliz porque cuando } \\
\text { estoy sembrando estoy } \\
\text { cuidando la naturaleza", } \\
\text { "he sentido como si } \\
\text { el árbol fuera un ser } \\
\text { humano", "si, alegre } \\
\text { porque voy a cuidar } \\
\text { mi medio ambiente”, } \\
\text { "Alegre y feliz", "si", "si } \\
\text { he sembrado árboles, me } \\
\text { he sentido como si fuera } \\
\text { amigo de la naturaleza, } \\
\text { también me he sentido } \\
\text { alegre, feliz, contento, } \\
\text { también me he sentido } \\
\text { como un granjero", } \\
\text { "me he sentido feliz, } \\
\text { alegre porque cuidamos } \\
\text { la naturaleza", "si, } \\
\text { porque en lo parques } \\
\text { es bonito sembrar", "si } \\
\text { he sembrado árboles, } \\
\text { se siente bien porque } \\
\text { nos hace sentir feliz y } \\
\text { me gusta sembrarlos } \\
\text { porque me gusta”, "me } \\
\text { siento alegre y contenta } \\
\text { y cuidando el medio } \\
\text { ambiente” "bien, eso me } \\
\text { pone feliz y contenta" } \\
\text { "feliz porque me gusta } \\
\text { sembrar semillas y } \\
\text { también árboles" "puedo } \\
\text { aprender muchas cosas } \\
\text { como sembrar el mango, } \\
\text { como quitarle el saco } \\
\text { para que crezca rápido". }\end{array}$ & 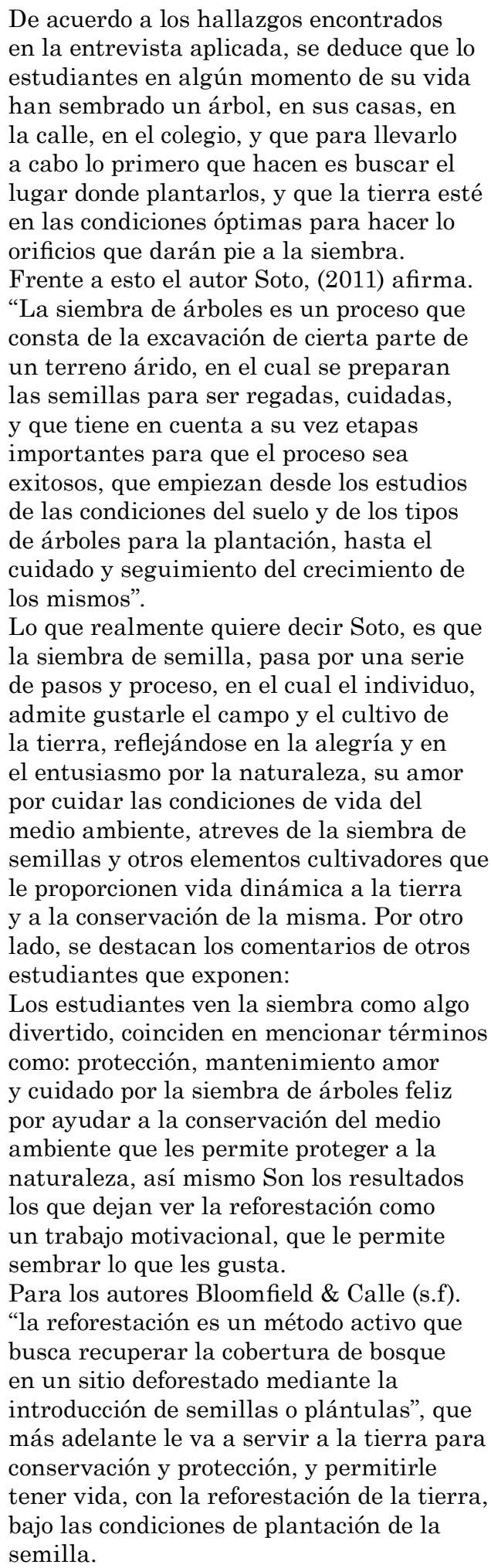 \\
\hline
\end{tabular}




$\begin{array}{ll} & \text { ¿Qué significa } \\ & \text { para ti } \\ \text { Reforestación } & \text { sembrar o } \\ \text { ambiental } & \text { plantar un } \\ & \text { árbol? }\end{array}$

¿Qué significa sembrar o árbol?
"Apostarle a la
naturaleza, ayudar
a la naturaleza y
el medio ambiente
por eso es que me
gusta", "crear más
vida, ayudar a la A la luz de lo expresado por los naturaleza”, "Crear oxígeno, ayudar a la naturaleza", "cuidar el ambiente natural, me siento feliz cuando siembro un árbol", "darle vida a la naturaleza para que nos de sombra”, "ayudar a la naturaleza”, "compasión porque le voy a dar vida a la naturaleza", "Bien porque nos ayuda a cuidar el medio ambiente", "me siento feliz y contenta sembrando un árbol”, "porque el medio ambiente estará seguro", "se siente bien cuidar el ambiente”, "tener oxígeno", "ser amigo de la naturaleza, amigo de las plantas, de las mariposas también”, "porque nos da medio ambiente", "ayudar al medio ambiente", "se siente bien”, darle oxígeno a la naturaleza”, "cuidar el medio ambiente", "me siento bien porque ayudo a cuidar el medio ambiente y a la naturaleza" Significa que para sembrar el mango lo primero que tienes que hacer es quitarle el saco para que crezca, si no le quitas el saco te dura un mes", "en el colegio, para la naturaleza”. estudiantes, éstos afirman que sembrar un árbol les ayuda a proteger a la naturaleza, contribuyen al cuidado del medio ambiente y a la generación de oxígeno. Resaltando además que una de las maneras de embellecer el colegio y sus alrededores es a través de la siembra.

La respuestas brindadas a través de la entrevista contribuyen a lo que afirma Reyes \& Gutiérrez (2010), quienes sostienen que es la siembra de árboles, la observación constante del procesos, sus cuidados, logra en los seres humanos una movilización de emociones, y grandes satisfacciones, porque indiscutiblemente se denota una apuesta al mejoramiento de la calidad de vida de la población.

Existe claridad de como plantar un árbol para genera resultados positivos si se realiza un procedimiento pertinente, tal como lo afirma Reyes \& Gutiérrez (2010) "La preparación del suelo, diseño, métodos, estrategias, los procesos de supervisión durante la actividad de reforestación, la protección, el mantenimiento, y los sistemas de evaluación que se tendrán en cuenta y que resulta factor primordial a la hora de iniciar una plantación y de mirar el éxito de la misma".

También se observa que es en el colegio, donde se tiene la necesidad de plantar, pues, es la reforestación una de las formas de manera activa de dar vida al área escolar por medio de la introducción de semillas o plántulas, es importante mirar el nivel de degradación del lugar al momento de plantar para así iniciar el crecimiento de una nueva vegetación y lo viable de una regeneración; para que el esfuerzo que se haga sea preciso para restaurar la diversidad de especies en el sitio. (Bloomfield \& Calle s.f). 


\begin{tabular}{|c|c|c|c|}
\hline $\begin{array}{l}\text { Programa } \\
\text { pedagógico }\end{array}$ & $\begin{array}{l}\text { ¿Crees tú que } \\
\text { sembrando } \\
\text { árboles ayuda } \\
\text { al medio } \\
\text { ambiente? Si- } \\
\text { no ¿Por qué? }\end{array}$ & 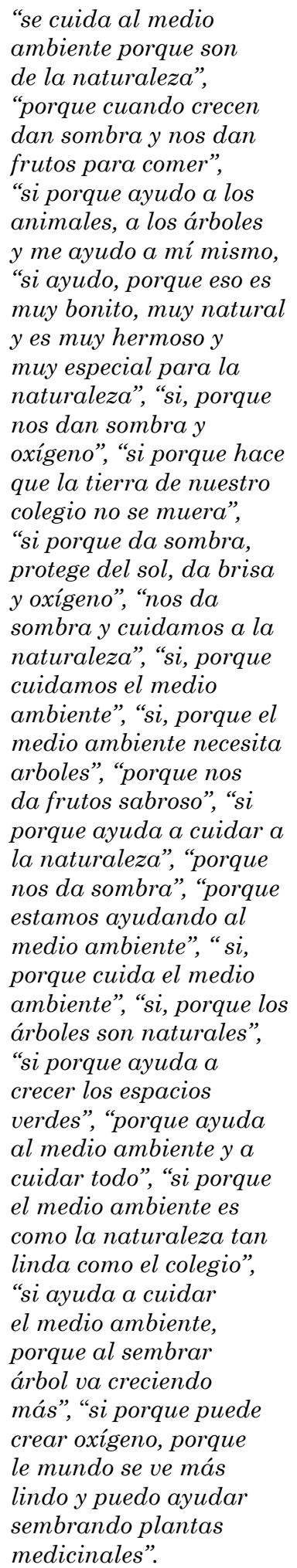 & $\begin{array}{l}\text { Las respuestas expuestas por } \\
\text { los estudiantes hacen notorio el } \\
\text { reconocimiento de la siembra como } \\
\text { actividad que, si busca el cuidado de } \\
\text { la naturaleza, su entorno, y además } \\
\text { de facilitar el crecimiento los espacios } \\
\text { ambientales. Un grupo significativo } \\
\text { también se alienan a la idea de que es } \\
\text { la siembra de árboles permite generan } \\
\text { sombras y frutos. } \\
\text { Herrera (2014) reafirma las conclusiones } \\
\text { explícitas por los estudiantes entrevistados } \\
\text { cuando expone que existen un conjunto } \\
\text { de sentimientos personales de normas u } \\
\text { obligación que conducen al establecimiento } \\
\text { de conductas pro ambientales, como la } \\
\text { siembra de árboles siempre y cuando } \\
\text { el individuo posea creencias relacionadas } \\
\text { con que las condiciones medioambientales } \\
\text { tienen efectos negativos para el mismo } \\
\text { individuo, su entorno y las demás especies } \\
\text { que lo habitan. } \\
\text { Otro de los hallazgos encontrados, fue } \\
\text { el manifestado por un estudiante, quien } \\
\text { afirman que la plantación de árboles } \\
\text { si favorece al medio ambiente por sus } \\
\text { beneficios a la salud, aspecto que cobra } \\
\text { gran importancia desde las pretensiones } \\
\text { del estudio. }\end{array}$ \\
\hline
\end{tabular}




\begin{tabular}{|c|c|c|c|}
\hline $\begin{array}{l}\text { Programa } \\
\text { pedagógico }\end{array}$ & $\begin{array}{l}\text { ¿En qué } \\
\text { lugar de } \\
\text { la sede } \\
\text { educativa } \\
\text { cree que } \\
\text { se deben } \\
\text { sembrar } \\
\text { árboles? }\end{array}$ & 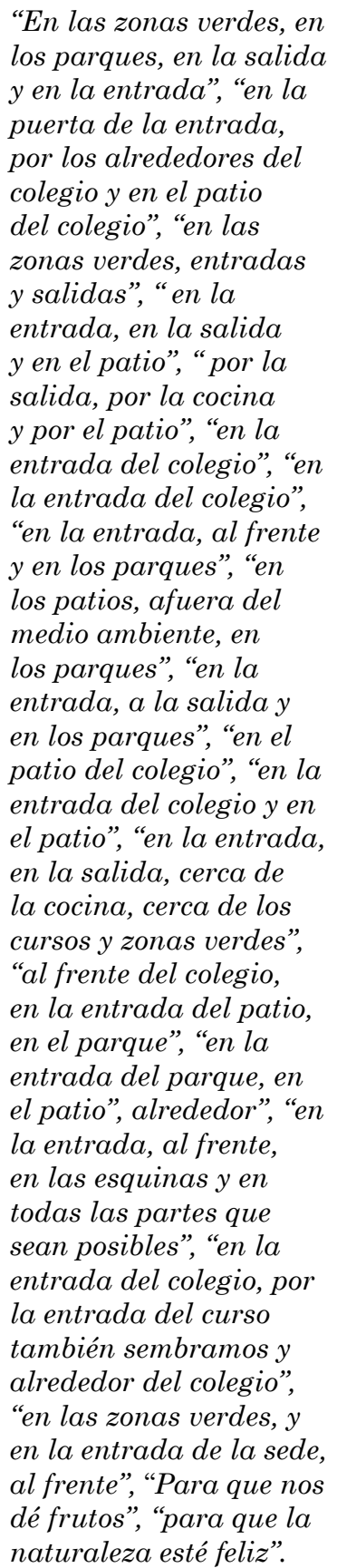 & $\begin{array}{l}\text { Los estudiantes reconocen los espacios en } \\
\text { donde se deben sembrar árboles, coincide } \\
\text { que, en todas las zonas verdes, entradas } \\
\text { y salidas del colegio, corredores, sin } \\
\text { embargo, hicieron un análisis de gran } \\
\text { relevancia, el parque que se encuentra a } 10 \\
\text { metros del colegio no cuenta con árboles, y } \\
\text { ven la necesidad de poder plantar. Esto se } \\
\text { articula a lo que expresa el Ministerio de } \\
\text { Educación de Chile (2008) en cuanto a que } \\
\text { la ejecución de un programa pedagógico } \\
\text { permite hacer seguimiento y gradación, a } \\
\text { partir de la observación y reconocimiento } \\
\text { de las necesidades de una problemática } \\
\text { estudiada, y la solución prevista desde los } \\
\text { actores directos. } \\
\text { Uno del propósito primordial es adecuar } \\
\text { de forma coherente las oportunidades } \\
\text { educativas, que no solo promueven la } \\
\text { enseñanza, sino además que potencian las } \\
\text { capacidades partiendo de las necesidades } \\
\text { del contexto. } \\
\text { Resulta también relevante anotar que } \\
\text { los resultados de las entrevistas a dos } \\
\text { estudiantes no expresan directamente } \\
\text { en qué lugares de la sede educativa se } \\
\text { debe sembrar árboles, sino que más bien } \\
\text { hacen hincapié a lo que generaría la } \\
\text { plantación, resaltando la necesidad de } \\
\text { realizar cambios actitudinales en los seres } \\
\text { humanos, que conduzcan a la generación } \\
\text { de modelos y patrones de conducta } \\
\text { más acordes con el cuidado del Medio } \\
\text { Ambiente partiendo desde su ciclo de } \\
\text { interacción vital y que conlleve a observar } \\
\text { resultados positivos a través de acciones } \\
\text { ambientalmente responsables. (Herrera, } \\
\text { 2014). }\end{array}$ \\
\hline
\end{tabular}

\section{Conclusiones}

Los hallazgos encontrados con anterioridad se enmarcan en el estudio denominado: Programa pedagógico para la reforestación ambiental en la EUN Policarpa Salavarrieta en el Departamento del Magdalena y generó las siguientes conclusiones: 
a. Los estudiantes y docentes reconocen la importancia conceptual y práctica de reforestar la sede educativa, por lo que se lograron apropiar de conceptos, técnicas y procedimientos propios del programa educativo, logrando transmitir nuevos conocimientos a los demás grupos de trabajo. En la fase inicial, el acompañamiento fue constante, pues los docentes también se vieron en la necesidad de realizar actividades de revisión documental, para la mayor apropiación y dominio del proceso de formación.

b. Por otro lado, el reconocimiento espacial, de zonas con necesidad de reforestar fue muy completo, son los estudiantes los primeros que distinguen lugares particulares (entrada, salida, corredores, parques etc.) que requieren de siembra de árboles, y el porqué de ellos.

c. Los procedimientos que se llevaron a cabo, constaron de análisis y estudio de las zonas, del suelo, y para ello tanto los maestros como los estudiantes revisaron las fases que requiere una plantación, generando conclusiones como la siguiente: Aunque son muchos los lugares en los que se debe plantar árboles, no todos cumplen con los criterios estipulados coherentemente para una siembra, y sobre todo para lograr los propósitos expuestos.

d. Son los estudiantes los que mencionaron arboles conocidos culturalmente en la región, y los resultados que ofrecen estos una vez crecen. En la descripción de la tipología de árboles, hubo desconocimiento en cuanto a las particularidades de ciertos árboles, en ciertos espacios dentro de la sede, sin embargo, se tomaron la tarea de estudiar tipos de árboles de acuerdo a los beneficios que se esperan obtener. e. Los árboles frutales y de gran expansión son los más opcionados según los estudiantes para ser plantados, por las descripciones y experiencias que han tenido en algún momento de su vida con ellos.

Finalmente, la consecución del programa pedagógico está en su fase de evaluación de impacto de acuerdo a las pretensiones iniciales, las etapas enmarcadas en el mismo han sido desarrolladas de manera adecuada. Los estudiantes reconocen la importancia de la reforestación, y los beneficios que ofrece a la naturaleza, a la institución y a la contribución de su desarrollo intelectual. De igual manera reconocen que la siembra de árbol va más allá de hacer orificios y plantar, identifican un estudio y análisis del suelo, la tipología de árboles de acuerdo al espacio, y su cuidado en el crecimiento.

\section{Referencias}

Alcaldía Mayor de Bogotá. (s.f.). Importancia de los árboles en el contexto urbano. Documento recuperado de: http://ambientebogota.gov.co/documents/10157/2066438/Importancia+ de+los+\%C3\%A1rboles+urbanos.pdf Alvarado, A., Guajardo, F. \& Devia, S. (2011). Manual de plantación de árboles en áreas urbanas. Legado. Vol. 1 (1). Santiago de Chile. Documento recuperado de: http://www.conaf.cl/ cms/editorweb/institucional/Manual_de_Plantacion_de_Arboles_ en_Areas_Urbanas.pdf.

Chávez. (s.f). Arborización. Universidad Nacional de Colombia. Documento recuperado de: https://arquitectura. medellin.unal.edu.co/images/imagenes/pdf/arborizacin.pdf 
Ecología Urbana: Experiencias en América Latina. Navarro-Sigüenza. Vol. 2. (1). Bloomfield, G. \& Calle, A. (s.f). Principios para la restauración de bosques tropicales: La reforestación. Elti. Vol. 1 (1).

Florez, N., Carvajal, A., Florez, L. \& Rincón A. (2012). Estrategias pedagógicas para transformar el entorno escolar de la institución educativa el quebradòn del Municipio de Rioblanco desde el trabajo social con estudiantes del grado once. (Tesis de Pregrado). Universidad del Tolima. Rioblanco, Tolima, Colombia.

Fundación Gondwana. (s.f.). Principales especies y características para la arborización de las zonas agrarias. Documento recuperado de: https://miradaverde.files.wordpress.com/2012/10/ funcion-de-arboles-bosques.pdf

Jaramillo, J. (1997). Guía para el diseño, construcción y operación de rellenos sanitarios manuales.

Línea Verde. La reforestación. Documento recuperado de: http://www.lineaverdeceutatrace.com/lv/consejos-ambientales/reforestemos/reforestemos.pdf

Lopez, J., Giraldo, N. \& Lopez, M. Proyecto de Educación Ambiental. (Proyecto de aula).

Marmolejo, L., Torres, P., Oviedo, E., Bedoya, E., Amezquita, C., Klinger, R., Albán, F, \& Díaz, L. (2009). Flujo de residuos: Elemento base para la sostenibilidad del aprovechamiento de residuos sólidos municipales. Ingeniería y competitividad. Vol. 1. (2). Universidad del Valle. Colombia.

Novo, M. (2009). La educación ambiental, una genuina educación para el desarrollo sostenible. Revista de Educación. Universidad Nacional de Educación a Distancia (UNED). Madrid, España.
PNUMA. (1990). Educación ambiental: modulo para la formación de profesores de ciencias y de supervisores para escuelas secundarias. Orelac. Santiago de Chile. Documento recuperado de: http://unesdoc.unesco.org/ images/0007/000714/071480so.pdf

Reyes, A. \& Gutierrez, J. (2010). Los servicios ambientales de la arborización urbana: Retos y aportes para la sustentabilidad de la Ciudad de Toluca. Quivera. Vol. 12(1). Universidad Autónoma de México. México.

Sandoval. C. (2002). Investigación cualitativa. ARFO. Bogotá Colombia. Documento recuperado de: https://panel.inkuba.com/sites/2/archivos/manual\%20colombia\%20cualitativo.pdf

Sauvé, L. (1999). La educación ambiental entre la modernidad y la posmodernidad: En busca de un marco educativo de referencia integrador. Tópicos. 1(2). México.

Sellitz, C. (1994). Metodología de la investigación. México: Editorial Prentice Hall Hispanoamericana.

Sheth, J. y Parvatiyar, A. (1995). Ecological imperatives and the role of marketing. En advances in environmental marketing. New developments in practice, theory and research. New York: The Hayworth press. Pp. 3-20.

Sheth, J. y Sidosia, R. (1999). Revisting marketing's lawlike generalizations. Journal of the academy of marketing science. 27(1). 71-87.

Sierra, R. (2007). Tesis Doctorales y trabajos de investigación científica. España: International Thompson editores Spain.

Soto, A. (2011). Las áreas verdes urbanas: Una alternativa para mejorar el microclima urbano. Cultura y Sociedad. 1(1) Documento recuperado de: Macgregor, I. \& Ortega, R. (2013). 
Stanley, L., Lasonde, K. y Weiss, J. (1996). The relationship between environmental issue involvement and environmentally-concious behavior: an exploratory study. Advances in consumer research. No. 23. Pp. 183-188.

Stern, P. (2000). Toward a coherent theory of environmentally significant behavior. Journal of Social Issues, 56(3), 407-424.

Stern, P. C., Dietz, T. \& Kalof, L. (1993). Value orientations, gender and environmental concern. Environment an Behavior, 25(3), 322-348.

Stern, P., Dietz, T. y Black, J. S. (1986). Support for environmental protection: The role of moral norms. Population and Environment, 8(1), 204-222.

Stern, P., Dietz, T. y Guagnano, G. (1995). The New Ecological Paradigm in Social-psychological context. Environment and Behavior, 27(6), 723-743.

Suárez, E. (1996). La participación ambiental como conducta altruista. En Ciudad y medio ambiente desde la experiencia humana. Mongrafies Psico/Socio/Ambientals, 10 (pp. 289-294). Barcelona: Universitat de Barcelona. Tamayo, M. (1993). El proceso de la investigación científica. Fundamentos de investigación con manual de evaluación de proyectos. Segunda edición. México: Editorial Limusa, S.A. Grupo Noriega Editores. 170 pp.
Toledo, V. y González, M. (2007). El metabolismo social: Las relaciones entre la sociedad y la naturaleza. Recuperado el 20 de noviembre de 2012 en: http://www.uv.mx/personal/ fpanico/files/2011/04/Toledo-y-Gonzalez-de-Molina-Metabolismo-social. pdf

Vining, J. \& Ebreo, A. (1992). Predicting Recycling Behavior from Global and Specific Environmental Attitudes and Changes in Recycling Opportunities. Journal of Applied Social Psychology, 22(20), 1580-1607.

Wagner, S. (1997) Understanding Green Consumer Behavior: A Qualitative Cognitive Approach. UK: Routledge.

Weigel, R. \& Weigel, J. (1978). Environmental concern: The development of a measure. Environment and Behavior, 10(1), 3-15.

Wood, D. (1991). Corporate social performance revisited. Academy of management review. 16(4). 691-718.

Zeithaml, C. y Zeithaml, V. (1984). Environmental management: Revising the marketing perspective. Journal of marketing. Vol. 48. Pp. 46-53.

Zikmund, W. y Stanton, W. (1971). Recycling solid wastes: A channelsof-distribution problem. Journal of marketing. 35. 34-39. 